\title{
Can 3D Shape of the Face Reveal your Age?
}

\author{
Baiqiang Xia ${ }^{1,3}$, Boulbaba Ben Amor ${ }^{2,3}$, Mohamed Daoudi ${ }^{2,3}$ and Hassen Drira ${ }^{2,3}$ \\ ${ }^{1}$ University Lille1, France. \\ ${ }^{2}$ Institut Mines-Telecom/Telecom Lille, France. \\ ${ }^{3}$ LIFL (UMR 8022 Lille 1/CNRS), France. \\ \{firstname.lastname\}@telecom-lille.fr
}

Keywords: Age estimation, 3D face, Dense Scalar Field, Random Forest Regression.

\begin{abstract}
Age reflects the continuous accumulation of durable effects from the past since birth. Human faces deform with time non-inversely and thus contains their aging information. In addition to its richness with anatomy information, 3D shape of faces could have the advantage of less dependent on pose and independent of illumination, while it hasn't been noticed in literature. Thus, in this work we investigate the age estimation problem from 3D shape of the face. With several descriptions grounding on Riemannian shape analysis of facial curves, we first extracted features from ideas of face Averageness, face Symmetry, its shape variations with Spatial and Gradient descriptors. Then, using the Random Forest-based Regression, experiments are carried out following the Leaving-One-Person-Out (LOPO) protocol on the FRGCv2 dataset. The proposed approach performs with a Mean Absolute Error (MAE) of 3.29 years using a gender-general test protocol. Finally, with the gender-specific experiments, which first separate the 3D scans into Female and Male subsets, then train and test on each gender specific subset in LOPO fashion, we improves the MAE to 3.15 years, which confirms the idea that the aging effect differs with gender.
\end{abstract}

\section{INTRODUCTION}

Face age estimation performs important social roles in human-to-human communication. Studies in cognitive psychology, presented as a review by (Rhodes, 2009), have discovered that human beings develop the ability of face age estimation naturally in early life, and can be fairly accurate in deciding the age or age group with a given face. These studies, based on subjective age estimation given to face image from human participants, have also found that multiple cues contribute to age estimation, including the holistic face features (like the outline of the face, face shape and texture, etc.), local face features (like the eyes, nose, the forehead, etc.) and their configuration (like the bilateral symmetry of the face (Clinton S. Morrison, 2011)). The aging process is a cumulative, uncontrollable and personalized slow process, influenced by intrinsic factors like the gene and gender, and extrinsic factors like lifestyle, expression, environment and sociality (Fu et al., 2010; Han et al., 2013). The appearance and anatomy of human faces changes remarkably with the progress of aging (Lanitis et al., 2002). The general pattern of the aging process differs in faces of different person (personalized or identity-specific), in faces of different age (age-specific), in faces of different gender (genderspecific), and in different facial components (Fu et al., 2010; Rhodes, 2009; Guo et al., 2009; Park et al., 2010; Guo et al., 2008b). Typically, the craniofacial growth (bone movement and growth) takes place during childhood, and stops around the age of 20, which leads to the re-sizing and re-distribution of facial regions, such as the forehead, eyes, nose, cheeks, lips, and the chin. From adulthood to old age, face changes mainly in the skin, such as the color changes (usually darker and with more color changes) and the texture changes (appearance of wrinkles). The shape changes of faces continues from adulthood to old age. With the droops and sags of facial muscle and skin, the faces are tend to be more a shape of trapezoid or rectangle in old faces, while the typical adult faces are more of a U-shaped or upside-down-triangle (Rhodes, 2009).

Automatic face age estimation is to label a face image with the exact age or age group objectively by machine. With the rapid advances in computer vision and machine learning, recently, automatic face age estimation have become particularly prevalent because of its explosive emerging and promising real-world applications, such as electronic customer relationship management, age- 
specific human-computer-interaction, age-specific access control and surveillance, law enforcement (e.g., detecting child-pornography, forensic), biometrics (e.g., age-invariant person identification (Park et al., 2010)), entertainment (e.g., cartoon film production, automatic album management), and cosmetology. Compared with human age estimation, automatic age estimation yields better performance as demonstrated in (Han et al., 2013). The performance of age estimation is typically measured by the mean absolute error (MAE) and the cumulative score (CS). The MAE is defined as the average of the absolute errors between the estimated age and the ground truth age, while the CS, proposed firstly by (Geng et al., 2007) in age estimation, shows the percentage of cases among the test set where the absolute age estimation error is less than a threshold. The CS measure is regarded as a more representative measure in relation with the performance of an age estimator (Lanitis, 2010).

As pointed in (Rhodes, 2009; Ramanathan et al., 2009), the earliest age estimation works used the mathematical cardioidal strain model, derived from face anthropometry that measures directly the sizes and proportions in human face, to describe the craniofacial growth. These approaches are useful for young ages, but not appropriate for adults. After this, abundant works exploiting 2D images have been published in the literature with more complex approaches. Different with the comprehensive surveys given by (Rhodes, 2009; Ramanathan et al., 2009), which categorized the literature concerning different aging modeling techniques, we represented the literature with the different ideas underlying these technical solutions. Based on the previous statements, we describe the face appearance as a function of multiple factors, including the age, the intrinsic factors (permanent factors like gene, gender, ethnicity, identity, etc.), and the extrinsic factors (temporary factors like lifestyle, health, sociality, expression, pose, illumination, etc.).

A. General aging patterns in face appearance. Essentially, face age estimation is to estimate the age of a subject by the aging patterns shown visually in the appearance. To analyze the appearance given in the face image is the basic ways to estimate the age. In the literature of age estimation, works were carried out with several different perceptions of the general aging patterns in face appearance. As aging exhibits similar patterns among different person, several approaches have been designed to learn the general public-level aging patterns in face appearance for age estimation. The most representative ones are the Active-AppearanceModel (AAM) based approaches, the manifold embedding approaches, and the Biologically-Inspired-
Feature (BIF) based approaches. The common idea underlying these approaches is to project a face (linearly or non-linearly) into a subspace, to have a low dimensional representation. Respectively, (i) (Lanitis et al., 2002; Lanitis et al., 2004) use an Active Appearance Model (AAM) based scheme for projecting face images linearly into a low dimensional space. The AAM was initially proposed by (Cootes et al., 1998), in which each face is represented by its shape and texture deviations to the mean face with a set of model parameters. Age estimation results with a quadratic regressor showed that the generic aging patterns work well for age estimation. Moreover,(Lanitis et al., 2004) illustrated that different face parameters obtained from training are responsible for different changes in lighting, pose, expression, and individual appearance. Considering that these parameters work well for age estimation, we can conclude that these face co-variants are influential in age estimation. (ii) The goal of manifold embedding approaches is to embed the original high dimensional face data in a lowerdimensional subspace by linear or non-linear projection, and take the embedding parameters as face representation. In the work of (Guo et al., 2008b; Guo et al., 2008a), the authors extracted age related features from 2D images with a linear manifold embedding method, named Orthogonal Locality Preserving Projections (OLPP). (Li et al., 2012) learned age manifold with both local preserving requirements and ordinal requirements to enhance age estimation performance (Wu et al., 2012) projected each face as a point on the Grassmann Manifold with the standard SVD method, then the tangent vector on these points of the manifold were taken as features for age estimation. (iii) Inspired by a feed-forward path theory in cortex for visual processing, (Guo et al., 2009) introduced the biologically inspired features (BIF) for face age estimation. After filtering an image with a Gabor filter and a standard deviation based filter consecutively, the obtained features are processed with PCA to generate lower-dimension BIF features. The results demonstrated the effectiveness and robustness of bioinspired features in encoding the generic aging patterns. Beyond the public-level aging patterns, there could be some less generic aging patterns when dealing with a subset of faces, such as a group of faces with high similarity, or a temporal sequence of face images for the same person. Based on the observation that similar faces tend to age similarly, (Lanitis et al., 2004; Lanitis et al., 2002) presented an appearancespecific strategy for age estimation. Faces are firstly clustered into groups considering their inter similarity, then training is performed on each group separately to learn a set of appearance-specific age estima- 
tors. Given a previously unseen face, the first step is to assign it to the most appropriate group, then the corresponding age estimator makes the age estimation. Experimental results showed that the group-level aging patterns are more accurate in age estimation compared with the generic-aging patterns. In case there is no similar enough face image for a testing face image in the database, (Lanitis et al., 2002) presented a weighted-appearance-specific approach which also yields fine performance. As different individual ages differently, (Geng et al., 2006; Geng et al., 2007) proposed the Aging-Pattern-Subspace (AGES), which studies the individual-level aging patterns from a temporal sequence of images of an individual ordered by time. For a test face, the aging pattern and the age is determined by the projection in the subspace that has the least reconstruction error. Experiments confirm that individual aging patterns contributes to age estimation. As different face components age differently, the component-level aging patterns are studied for age estimation. (Suo et al., 2010) represented faces with a hierarchical And-Or Graph. Face aging is then modeled as a Markov process on the graphs and the learned parameters of the model are used for age estimation. They found that the forehead and eye regions are the most informative for age estimation, which is also supported by discoveries of (Han et al., 2013) using the BIF features.

B. Considering the intrinsic/extrinsic factors in facial aging. As stated at the beginning of this introduction, the appearance of face is influenced by intrinsic factors like the gene, gender, and extrinsic factors like lifestyle, expressions, environment and sociality (Fu et al., 2010; Han et al., 2013). Several studies have given consideration of the influences of these factors in age estimation with enhanced age estimation performance reported. Specifically, thinking that faces age differently in different age, age-specific approaches are adopted by (Lanitis et al., 2004), where age estimation is obtained by using a global age classifier first, then adjusted the estimated age by a local classifier which operates within a specific age range. Similarly, (Guo et al., 2008b; Guo et al., 2008a) proposed a Locally Adjusted Robust Regressor (LARR) for age estimation, which begins with a SVR-based global age regression, then followed by a local SVMbased classification that adjusts the age estimation in a local age range. All of these age-specific approaches have achieved better performance compared with their corresponding approaches without local adjustment. Considering that different gender ages differently with age (Ramanathan et al., 2009; Guo et al., 2008a), (Ueki et al., 2010; Ramanathan et al., 2009; Guo et al., 2008a; Lakshmiprabha et al., 2011) carried out age estimation on male and female groups separately. Considering the individual lifestyle, (Lanitis et al., 2002) encoded this information together with facial appearance in age estimation, and demonstrated that the importance of lifestyle in determining the most appropriate aging function of a new individual. (Ueki et al., 2010) gave weights to different lighting conditions for illumination-robust face age estimation. (Li et al., 2012) gave consideration of the feature redundancy and used feature selection to enhance age estimation. As stated before, in the childhood, face deformation mainly takes the form of craniofacial growth with facial features re-sized and re-distributed. From adulthood to old age, with the droops and sags of facial muscle and skin, the old faces usually deform to a trapezoid or rectangle shape from a typically U-shaped or upside-down-triangle in adult face (Rhodes, 2009). Another significant shape deformation is the introduction of facial wrinkles with aging. While, given the fact that face shape deforms significantly with age in three dimensions, and given the robustness of 3D face scans to illumination and poses compared with 2D face images, all the previous works in the literature used $2 \mathrm{D}$ face datasets for age estimation, no work has been done concerning the 3D face. Thus, in this work, we introduce the investigation of age estimation with $3 \mathrm{D}$ face scans. The rest of the paper is organized as follows: in section 2 , we present an overview of our methodology and summarize the main contributions; in section $\mathbf{3}$, we explain our methodology of features extraction from the 3D faces based on an Riemann framework; in section 4, we detail the regression strategy for age estimation using Random Forest; experimental results and their discussion are presented in section $\mathbf{5}$ while section $\mathbf{6}$ comes to the conclusion of this work.

\section{METHODOLOGY AND CONTRIBUTION}

From the analysis above, it emerges that most of the existing works study age estimation with aging patterns chosen at a specified level and some aging factors enrolled for enhancement. As far as we concern, all these works are based on 2D images, no work concerning 3D face scans has been attached to age estimation. Thus, we introduce in the present work a new study of 3D-based face age estimation to the domain. In our approach, we consider the publiclevel aging patterns and gender factor for age estimation. First, we extract four types of Dense Scalar Field (DSF) features from each pre-processed face, namely the Average DSF, the Symmetry DSF, the Spatial DSF and the Gradient DSF. These DSFs are derived from 
different face perception ideas and their computation is grounding on Riemannian shape analysis of facial curves. Then we perform age estimation using Random Forest Regression on each type of DSFs with two protocols: one experiment on DSFs of the whole dataset directly and the other experiments on male and female DSFs separately. We have also designed a simple result-level fusion with different type of the DSFs, to see if the performance improves with all these face perception ideas combined.

In summary, the main contributions of this work are as follows. First, as far as we know, this is the first work in 3D-based age estimation. Although 3D face growth has been notice for a long time (Mark and Todd, 1983; Bruce et al., 1989), no work has been reported to 3D face age estimation. Secondly, in this work, we introduce four different perspectives of faces perception for face representation. With the Dense Scalar Field features, we have obtained significant accuracy with each of the perspectives, compared with typical 2D-based age estimation performance. Last but not the least, we have enhanced the age estimation performance by experimenting on the scans of each gender separately, which confirms that the sexual dimorphism exists in terms of face aging patterns. We have also enhanced the performance by a simple late fusion rule of the four descriptors.

\section{FEATURE EXTRACTION}

As mentioned earlier, we adopt the Dense Scalar Field features in our approach. Based on pairwise shape comparison of curves, the Dense Scalar Field (DSF) grounding on Riemannian shape analysis (Drira et al., 2012) (Drira et al., 2013) is capable for capturing the local shape deformation between facial curves. Formally, for any curve in the space of $\mathbb{R}^{3}, \beta$ : $I \rightarrow \mathbb{R}^{3}$, where $I=[0,1]$, it is first represented mathematically by the square-root velocity function $q(t)$, according to: $q(t)=\frac{\dot{\beta}(t)}{\sqrt{\|\dot{\beta}(t)\|}}$ (Srivastava et al., 2011). With the $\mathbb{L}^{2}$ norm $\|\cdot\|$ scaled to 1 , the space of such functions: $\mathcal{C}=\left\{q: I \rightarrow \mathbb{R}^{3},\|q\|=1\right\} \subset \mathbb{L}^{2}\left(I, \mathbb{R}^{3}\right)$ becomes a Riemannian manifold with the $\mathbb{L}^{2}$ metric on its tangent spaces. Since $\|q\|=1, C$ is a also a Hypersphere in the Hilbert space $\mathbb{L}^{2}\left(I, \mathbb{R}^{3}\right)$. Given two curves $\beta_{1}$ and $\beta_{2}$, they are first represented by the square-root velocity function, then unified to $q_{1}$ and $q_{2}$ with $\|q\|=1$. The geodesic path $\psi^{*}$ between $q_{1}, q_{2}$ on the manifold $C$ is given by the minor arc of great circle connecting them on this Hypersphere, $\psi^{*}:[0,1] \rightarrow \mathcal{C}$ given by (1),

$$
\psi^{*}(\tau)=\frac{1}{\sin (\theta)}\left(\sin ((1-\tau) \theta) q_{1}+\sin (\theta \tau) q_{2}\right)
$$

where $\theta=d_{C}\left(q_{1}, q_{2}\right)=\cos ^{-1}\left(\left\langle q_{1}, q_{2}\right\rangle\right)$ is the angle between $q_{1}$ and $q_{2}$. The tangent vector field on this geodesic $\dot{\psi}^{*}:[0,1] \rightarrow T_{\psi}(\mathcal{C})$ is then given by (2):

$$
\dot{\psi}^{*}=\frac{d \psi^{*}}{d \tau}=\frac{-\theta}{\sin (\theta)}\left(\cos ((1-\tau) \theta) q_{1}-\cos (\theta \tau) q_{2}\right)
$$

Knowing that on a geodesic path, the covariant derivative of its tangent vector field is equal to 0 . Thus, $\left.\dot{\psi}^{*}\right|_{\tau=0}$ is sufficient to represent this vector field. Accordingly, (2) becomes:

$$
\left.\dot{\psi}^{*}\right|_{\tau=0}=\frac{\theta}{\sin (\theta)}\left(q_{2}-\cos (\theta) q_{1}\right)
$$

With the magnitude of $\dot{\psi}_{\alpha}{ }^{*}$ at each all the $\mathrm{N}$ indexed points of the curve, we build a Dense Scalar Field (DSF),$V=\left\{\left\|\left.\dot{\psi}^{*}\right|_{(\tau=0)}(k)\right\|, k=1,2,3, . ., N\right\}$, which quantifies the shape difference between two curves.

In our approach, the raw 3D face scans are first pre-processed for hole-filling, cropping, smoothing and pose normalization, and then represented by a set of parameterized radial curves emanating from the nose tip of the preprocessed face denoted with $\boldsymbol{S}$. The radial curve that makes an clockwise angle of $\alpha$ with the radial curve which passes through the forehead $\left(\beta_{0}\right)$ is denoted as $\beta_{\alpha}$, and the neighbor curve of $\beta_{\alpha}$ that has an angle increase of $\Delta \alpha$ is denoted as $\beta_{\alpha+\Delta \alpha}$. Such representation can be seen as a approximation of the preprocessed face $\boldsymbol{S}$. To extract the DSF features, one need to first define the correspondence of curves in pair-wise shape comparison. With four different perspectives from face perception, we define four different types of correspondence in pairwise shape comparison, which results into four different types of DSF features with all the radial curves considered in a face, namely the Symmetry DSF, the Averageness DSF, the Spatial DSF and the Gradient DSF. Figure 1 gives an illustration of these DSF features. The Symmetry DSF shown in sub-figure (a) captures the deformation between a pair of bilateral symmetrical radial curves $\left(\beta_{\alpha}^{S}\right.$ and $\beta_{2 \pi-\alpha}^{S}$ ) in a preprocessed face $S$. The Symmetry DSF conveys the idea that the bilateral facial symmetry loses with age. The Averageness DSF shown in sub-figure (b) compares a pair of curves with the same angle index from a preprocessed face $\beta_{\alpha}^{S}$ and an average face template $\beta_{\alpha}^{T}$. The average face template $\boldsymbol{T}$ (as presented in subfigure (b)) is defined as the middle point of geodesic deformation path from a representative male scan to a representative female scan. The Averageness DSF represents the idea that faces become more personalized and thus deviates more from the average face shape with age. The Spatial DSF shown in sub-figure (c) captures the deformation of a curve $\beta_{\alpha}$ to one ref- 
(a)

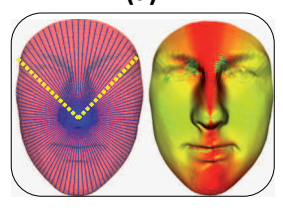

(b)

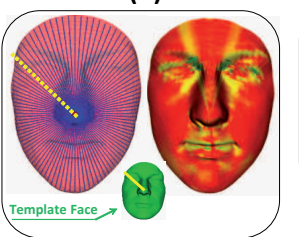

(c)

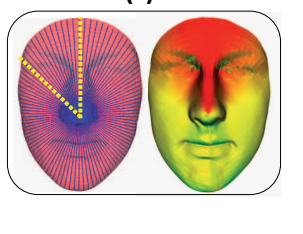

(d)

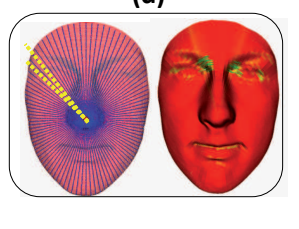

Figure 1: Illustrations of different DSFs on preprocessed face $S$. (a) Symmetry DSF: the DSF from radial curve $\beta_{\alpha}^{S}$ to its bilateral symmetrical curve $\beta_{2 \pi-\alpha}^{S}$; (b) Averageness DSF: DSF from radial curve $\beta_{\alpha}^{S}$ in a preprocessed face to radial curve $\beta_{\alpha}^{T}$ in an average face template (with the same angle index $\alpha$ ); (c) Spatial DSF: DSF from radial curve $\beta_{\alpha}^{S}$ to the middle radial curve $\beta_{0}^{S}$ in the forehead; (d) Gradient DSF: DSF from radial curve $\beta_{\alpha}^{S}$ to its neighbor curve $\beta_{\alpha+\Delta \alpha}^{S}$.

erence radial curve $\beta_{0}$ in the forehead in a preprocessed face $S$. As $\beta_{0}$ is the most rigid curve in the face, the Spatial DSF can be perceived as the cumulative deformation from the most rigid part of the face. The Gradient DSF shown in sub-figure (d) captures the deformation between a pair of neighbor curves $\left(\beta_{\alpha}^{S}\right.$ and $\beta_{\alpha+\Delta \alpha}^{S}$ ) in a preprocessed face $S$. In contrast with the Spatial DSF, the Gradient DSF can be viewed as a representation of local deformation on the face. In each sub-figure of Figure 1, the left part shows the extracted radial curves in the face and correspondence for curve comparison, the right part shows the corresponding DSF features as color-map on the face, where on each face point, the hotter the color, the lower of the DSF magnitude.

\section{RANDOM FOREST REGRESSION}

Age estimation can be considered as a classification problem, when each age is taken as a class label. On the other hand, age estimation can also be considered as a regression problem, since the age could be interpreted as continuous value. Note that there are only 15 subjects of more than 40 years old in FRGCv2, the number of faces is too small to train classifiers for those ages. Thus, in our approach, we take the age estimation as a regression problem. Similar reason has been used by (Guo et al., 2009) for choosing the regression strategy for age estimation on the FG-net dataset, where the images from old subjects are also rare. As summarized by (Criminisi and Shotton, 2013), the regression task is, given a labeled set of training data, learning a general mapping which associates previously unseen, independent test data points with their dependent continuous output prediction. In the work of (Montillo and Ling, 2009), Random Forest regression has demonstrated nice age estimation performance (3.43 MAE) in LOPO experiments for the young age subset of the FG-net dataset. As far as we concern, no studies have investigated the age estimation performance of Random Forest with the overall age distribution. Thus, we adopt the Random Forest in our regression experiments to demonstrate its capability in age estimation. Technically, Random Forest is an ensemble learning method that grows many classification trees $t \in\left\{t_{1}, . ., t_{T}\right\}$. To estimate age from a new face from an input vector (DSFbased feature vector $v=V_{\alpha}^{k}$ ), each tree gives a regression result and the forest takes the average of estimated ages as the final result. In the growing of each tree, two types of randomness are introduced consecutively. Firstly, a number of $\boldsymbol{N}$ instances are sampled randomly with replacement from the original data, to make the training set. Then, if each instance comprises of $\boldsymbol{M}$ input variables, a constant number $\boldsymbol{m}(\boldsymbol{m}<<\boldsymbol{M})$ is specified. At each node of the tree, $\boldsymbol{m}$ variables are randomly selected out of the $\boldsymbol{M}$ and the best split on these $\boldsymbol{m}$ variables is used to split the node. The process goes on until the tree grows to the largest possible extent without pruning, where the resulted subsets of the node are totally purified in label.

\section{EXPERIMENTS}

Our experiments are carried out with Random Forest Regression on FRGCv2 dataset. The FRGCv2 dataset was collected by researchers from the University of Notre Dame and contains 4007 3D nearfrontal face scans of 466 subjects, where 203 are female and 263 are male (Phillips et al., 2005). The age of subjects ranges from 18 to 70 , with $92.5 \%$ in the 18-30 age group. Our experiments are performed with the 466 earliest scans of each subject in FRGCv2. With the 466 earliest scans, we design two experiment protocols. The first protocol, named Gender-General-Protocol (GGP), experiments on the 466 scans directly with Random Forest Regression. While the second protocol, named Gender-SpecificProtocol (GSP), separates the 466 scans into male group and female group first, and then performs ex- 
periments on each group separately with Random Forest Regression. For all the two protocols, experimental results are generated using the Leave-OnePerson-Out (LOPO) cross-validation strategy, where each time one scan of the concerning data (all 466 scans or scans of each gender) is used as testing face once, with the rest the scans used in training. Thus, there are altogether 466 experiments in the crossvalidation in each protocol, and each scan is tested equally only once.

\subsection{Gender-general experiment}

As described above, with the Gender-GeneralProtocol (GGP), we perform Leave-One-Person-Out cross-validation experiments directly with the 466 earliest scans of FRGCv2 dataset for each descriptor. Each time one scan is picked out for testing and the rest 465 scans are used for training. Table 1 shows the experimental results as the mean of the absolute error between the truth and the estimated age for each tested scan in corresponding age group. By taking the minimum value of the estimated ages given by the four descriptors as the age estimation result, we have also obtained the fusion results, as shown also in Table 1. From this table, we observe that we achieve a minimum overall mean absolute error (MAE) about 3.7 years by the Averageness and Spatial DSFs. For the other three descriptors, the overall mean absolute errors are a little higher, while all of them are under 4 years. Thus, from the perception of the overall mean absolute errors, we find that our approaches with all of the four descriptors are effective in age estimation. Moreover, when we go inside of the details of these results for each age group, we find that the age estimation performance declines significantly with aging. We assume that the big decrease of the number of scans in aged groups (from about 200 to about 20) accounts largely for this performance decline. From the same table, we also observe that the fusion method, which takes the minimum of the estimated ages concerning each of the four descriptors, yields a better overall mean absolute error of 3.29 years. It means that the age related cues in these descriptors are different and complimentary in age estimation. When going inside of the detail of the fusion result for each age group, we find the enhancement of overall performance is mainly coming from young age groups. It is probably due to the fact that for young age groups, more scans are available in training for each descriptor. Thus the estimation results from each descriptor for young age groups are less biased for making the fusion decision.

Figure 2 shows the experimental results of Gender-General-Protocol by cumulative scores for the four descriptors. The $\mathrm{x}$-axis is the level of Mean
Table 1: Age estimation results for different age groups with the Gender-General-Protocol. (MAE:Mean Absolute Error; AVR: Averageness; SYM: symmetry; GRA: gradient; SPA: spatial; MIN: minimum rule for fusion.)

\begin{tabular}{|l|l|l|l|l|l|l|}
\hline $\begin{array}{l}\text { Age } \\
\text { group }\end{array}$ & $\begin{array}{l}\text { MAE } \\
\text { AVR }\end{array}$ & $\begin{array}{l}\text { MAE } \\
\text { SYM }\end{array}$ & $\begin{array}{l}\text { MAE } \\
\text { GRA }\end{array}$ & $\begin{array}{l}\text { MAE } \\
\text { SPA }\end{array}$ & $\begin{array}{l}\text { Fuse } \\
\text { MIN }\end{array}$ & $\begin{array}{l}\sharp \text { of } \\
\text { scans }\end{array}$ \\
\hline \hline$\leq 20$ & 3.48 & 3.43 & 3.77 & 3.30 & 2.20 & 185 \\
\hline$(20,30]$ & 2.18 & 2.58 & 2.32 & 2.38 & 1.98 & 246 \\
\hline$(30,40]$ & 9.99 & 7.60 & 10.05 & 8.92 & 9.18 & 20 \\
\hline$>40$ & 24.82 & 23.66 & 24.56 & 25.36 & 25.75 & 15 \\
\hline \hline Overall & $\mathbf{3 . 7 6}$ & $\mathbf{3 . 7 9}$ & $\mathbf{3 . 9 4}$ & $\mathbf{3 . 7 6}$ & $\mathbf{3 . 2 9}$ & $\mathbf{4 6 6}$ \\
\hline
\end{tabular}

Absolute Error, which represents the mean of the absolute age error (between the truth and estimated age of scan) over the 466 scans. The y-axis show the cumulative score of accuracy by percentage of acceptance. Thus, a point $(a, b)$ on the curve shows, with a Mean Absolute Error tolerance of $a$ years, it achieves an acceptance of $b$ percent. We have also captured the fusion result in the same figure by cumulative scores. From Figure 2, we observe that with a Error Level of 5 years, we achieve an acceptance of more than $75 \%$ over the 466 scans; when the Error Level is 10 years, the cumulative score of acceptance increases to more than $90 \%$. We also observe that the fusion result is significantly higher compared with the result of each individual descriptor. From these observations, we claim again that our approach concerning all these descriptors are comparably effective in age estimation, and the result-level fusion of these descriptors enhances the age estimation performance.

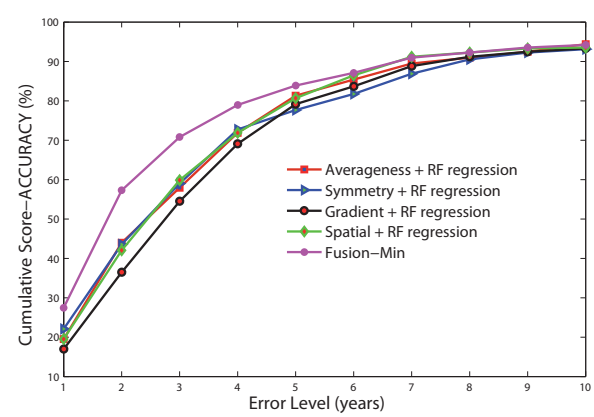

Figure 2: Age regression results in Leave-One-Person-Out experiment with the Gender-General-Protocol.

\subsection{Gender-specific experiment}

Gender and age are natural co-variates in human face. In (Samal et al., 2007), Ashok Samal et al. statistically confirm that sexual dimorphism is strong and widespread among face features, and find out the degree of dimorphism changes as a function age (e.g., the average age at which the sexual dimorphism be- 
comes more significant is around 13). Thus, the face aging effect is considerably different with different gender. In the experiments considering the GenderSpecific-Protocol (GSP), we first separate the 466 earliest scans of FRGCv2 into male group and female group, then we perform Leave-One-Person-Out cross-validation experiments on male scans and female scans separately for each descriptor. As in the GGP experiments, each time we take one scan in testing and the rest scans in training. The final results for each descriptor are generated by statistically merging the results from each gender.

Table 2: Results for different age groups with the GenderSpecific-Protocol. (MAE:Mean Absolute Error; AVR: Averageness; SYM: symmetry; GRA: gradient; SPA: spatial.)

\begin{tabular}{|l|l|l|l|l|l|l|}
\hline $\begin{array}{l}\text { Age } \\
\text { group }\end{array}$ & $\begin{array}{l}\text { MAE } \\
\text { AVR }\end{array}$ & $\begin{array}{l}\text { MAE } \\
\text { SY }\end{array}$ & $\begin{array}{l}\text { MAE } \\
\text { GRA }\end{array}$ & $\begin{array}{l}\text { MAE } \\
\text { SPA }\end{array}$ & $\begin{array}{l}\text { Fuse } \\
\text { MIN }\end{array}$ & $\begin{array}{l}\sharp \text { of } \\
\text { scans }\end{array}$ \\
\hline \hline$\leq 20$ & 3.25 & 3.38 & 3.46 & 3.19 & 2.14 & 185 \\
\hline$(20,30]$ & 2.03 & 2.16 & 2.14 & 2.18 & 2.04 & 246 \\
\hline$(30,40]$ & 8.97 & 8.52 & 9.18 & 8.81 & 10.43 & 20 \\
\hline$>40$ & 20.81 & 22.59 & 21.32 & 22.22 & 24.05 & 15 \\
\hline \hline Overall & $\mathbf{3 . 4 2}$ & $\mathbf{3 . 5 7}$ & $\mathbf{3 . 5 8}$ & $\mathbf{3 . 5 1}$ & $\mathbf{3 . 1 5}$ & $\mathbf{4 6 6}$ \\
\hline
\end{tabular}

Table 2 shows the experimental results as the mean of the absolute error between the truth and the estimated age for each tested scan in corresponding age group. From Table 2, we observe that for all the four descriptors, we always achieve better overall performance with GSP. We also achieve better results in each age group with all these descriptors, except for the symmetry descriptor in the $(30,40]$ age group. With these observations, which indicate that the Gender-Specific-Protocol outperforms the Gender-General-Protocol in age estimation, we confirm the claims in (Samal et al., 2007), that faces of different gender convey considerably different morphology of aging. Moreover, the overall fusion result outperforms again the result of each descriptor in the GSP experiments, and also the overall fusion result in the GGP experiments. It shows again that the resultlevel fusion of these descriptors can enhance the age estimation performance.

Figure 3 makes a further comparison between the GGP and GSP experiments, with the cumulative scores for each gender and for each descriptor in these two type of experiments. From Figure 3, we observe that, only except for the beginning part of result with the female group and symmetry descriptor, the experimental results are always significantly higher for both male and female groups in the GSP experiments for all the descriptors. That is to say, although trained with less data, the GSP experiments have the advantage of giving better age regression results. One prob-
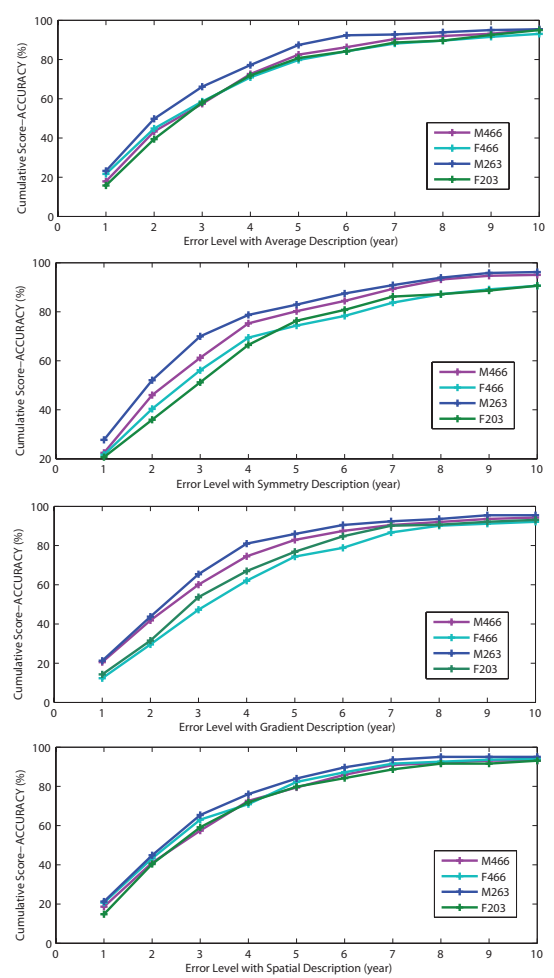

Figure 3: Comparison of results from the Gender-GeneralProtocol (GGP) and the Gender-Specific-Protocol (GSP) for each gender. (M-gender-general: male group in GGP experiments; F-gender-general: female group in GGP experiments; M-gender-specific: male group in GSP experiments; F-gender-specific: female group in GSP experiments.)

able explanation for this observation is that, in the GSP experiments, the regression results do not suffer the influence from the scans in the other gender, which conveys a significantly different aging morphology. With Figure 3, we further confirm that the aging effect differs with gender.

\section{CONCLUSIONS}

In this paper, we presented the first work in age estimation based on 3D facial scans. Our approach uses the DSF features extracted from 3D face from four different perspectives of face perception. Following the Leave-One-Person-Out experimental setting when using the Random Forest Regression strategy, we have achieved comparable age estimation results with all the four descriptions. And with the age estimation results improved in their fusion, we have confirmed that the four perspectives produce complimentary information for age estimation. By investigating the age estimation separately on Female and 
Male subsets, we have achieved better age estimation results, which justifies that the general aging effect of face differs considerably with gender.

\section{ACKNOWLEDGMENTS}

This work was supported by the ANR through the 3D Face Analyzer project under the contract ANR 2010 INTB 030101 and by the Chinese Scholarship Council (CSC) to Baiqiang Xia.

\section{REFERENCES}

Bruce, V., Burton, M., Doyle, T., and Dench, N. (1989). Further experiments on the perception of growth in three dimensions. 46(6):528-536.

Clinton S. Morrison, Benjamin Z. Phillips, J. T. C. S. R. S. H. O. T. (2011). The relationship between age and facial asymmetry. In http://meeting.nesps.org/2011/80.cgi.

Cootes, T. F., Edwards, G. J., and Taylor, C. J. (1998). Active appearance models. In Computer Vision ECCV98, pages 484-498.

Criminisi, A. and Shotton, J. (2013). Regression forests. In Decision Forests for Computer Vision and Medical Image Analysis, pages 49-58.

Drira, H., Ben Amor, B., Daoudi, M., Srivastava, A., and Berretti, S. (2012). 3D dynamic expression recognition based on a novel deformation vector field and random forest. In ICPR, pages 1104-1107.

Drira, H., Ben Amor, B., Srivastava, A., Daoudi, M., and Slama, R. (2013). 3d face recognition under expressions, occlusions, and pose variations. IEEE Trans. Pattern Anal. Mach. Intell., 35(9):2270-2283.

Fu, Y., Guo, G., and Huang, T. S. (2010). Age synthesis and estimation via faces: A survey. In IEEE Transactions on Pattern Analysis and Machine Intelligence, volume 32, pages 1955-1976.

Geng, X., Zhou, Z.-H., and Smith-Miles, K. (2007). Automatic age estimation based on facial aging patterns. 29(12):2234-2240.

Geng, X., Zhou, Z.-H., Zhang, Y., Li, G., and Dai, H. (2006). Learning from facial aging patterns for automatic age estimation. In Proceedings of the 14th annual ACM international conference on Multimedia, pages 307-316. ACM.

Guo, G., Fu, Y., Dyer, C. R., and Huang, T. S. (2008a). Image-based human age estimation by manifold learning and locally adjusted robust regression. 17(7):1178-1188.

Guo, G., Fu, Y., Huang, T. S., and Dyer, C. R. (2008b). Locally adjusted robust regression for human age estimation. In Applications of Computer Vision, 2008. WACV 2008. IEEE Workshop on, pages 1-6. IEEE.

Guo, G., Mu, G., Fu, Y., and Huang, T. S. (2009). Human age estimation using bio-inspired features. In Computer Vision and Pattern Recognition, 2009. CVPR 2009. IEEE Conference on, pages 112-119.
Han, H., Otto, C., and Jain, A. K. (2013). Age estimation from face images: Human vs. machine performance. In The 6th IAPR International Conference on Biomet$\operatorname{rics}(I C B)$.

Lakshmiprabha, N., Bhattacharya, J., and Majumder, S. (2011). Age estimation using gender information. In Computer Networks and Intelligent Computing, pages 211-216.

Lanitis, A. (2010). Facial age estimation. In Scholarpedia, volume 5, page 9701.

Lanitis, A., Draganova, C., and Christodoulou, C. (2004). Comparing different classifiers for automatic age estimation. In Systems, Man, and Cybernetics, Part B: Cybernetics, IEEE Transactions on, volume 34, pages 621-628. IEEE.

Lanitis, A., Taylor, C. J., and Cootes, T. F. (2002). Toward automatic simulation of aging effects on face images. In Pattern Analysis and Machine Intelligence, IEEE Transactions on, volume 24, pages 442-455. IEEE.

Li, C., Liu, Q., Liu, J., and Lu, H. (2012). Learning ordinal discriminative features for age estimation. In Computer Vision and Pattern Recognition (CVPR), 2012 IEEE Conference on, pages 2570-2577.

Mark, L. S. and Todd, J. T. (1983). The perception of growth in three dimensions. 33(2):193-196.

Montillo, A. and Ling, H. (2009). Age regression from faces using random forests. In Image Processing (ICIP), 2009 16th IEEE International Conference on, pages 2465-2468. IEEE.

Park, U., Tong, Y., and Jain, A. K. (2010). Age-invariant face recognition. 32(5):947-954.

Phillips, P. J., Flynn, P. J., Scruggs, T., Bowyer, K. W., Chang, J., Hoffman, K., Marques, J., Min, J., and Worek, W. (2005). Overview of the face recognition grand challenge. In Computer vision and pattern recognition, CVPR 2005, volume 1, pages 947-954.

Ramanathan, N., Chellappa, R., and Biswas, S. (2009). Computational methods for modeling facial aging: A survey. 20(3):131-144.

Rhodes, M. G. (2009). Age estimation of faces: a review. In Appl. Cognit. Psychol, volume 23, pages 1-12.

Samal, A., Subramani, V., and Marx, D. (2007). Analysis of sexual dimorphism in the human face. In Journal of Visual Communication and Image Representation, pages 453-463.

Srivastava, A., Klassen, E., Joshi, S. H., and Jermyn, I. H. (2011). Shape analysis of elastic curves in euclidean spaces. In Pattern Analysis and Machine Intelligence, volume 33, pages 1415-1428. IEEE.

Suo, J., Zhu, S.-C., Shan, S., and Chen, X. (2010). A compositional and dynamic model for face aging. volume 32, pages 385-401.

Ueki, K., Sugiyama, M., and Ihara, Y. (2010). Perceived age estimation under lighting condition change by covariate shift adaptation. In Pattern Recognition (ICPR), 2010 20th International Conference on, pages 34003403. IEEE.

Wu, T., Turaga, P., and Chellappa, R. (2012). Age estimation and face verification across aging using landmarks. volume 7 , pages $1780-1788$. 